\section{Christoph Bachmann}

\section{Einleitung}

Weltweit leiden Millionen von Frauen unter menopausalen Beschwerden. Lange Zeit galt eine Therapie dieser klimakterischen Beschwerden mit Hormonen (engl. «hormone replacement therapy» (HRT)) als Goldstandard. Die Problematik des Zusammenhangs dieser Hormontherapie mit einem erhöhten Risiko von Mammakarzinomen rückte die Diskussion über Alternativen bzw. natürliche Behandlungen von menopausalen Beschwerden in das Zentrum des Interesses. In Kreisen der Komplementärmedizin wurden von Ärzten, Hebammen und Naturheilpraktikern immer häufiger Präparate aus verschiedenen Arzneipflanzen eingesetzt. In der Folge werden einige dieser Anwendungen beschrieben und die Studienlage aufgezeigt.

\section{Arzneipflanzen}

Tabelle 1 stellt eine Übersicht von Arzneipflanzen dar, die gegen menopausale Symptome eingesetzt werden. Hier wird deutlich, dass Extrakte aus Cimicifuga racemosa (Traubensilberkerze) mit Abstand die beste Studienlage besitzen und als einzige der aufgeführten Arzneipflanzen in der Schweiz registrierte Arzneipräparate vorweisen kann, die zum Teil auch von der Grundversicherung erstattet werden.

\section{Aus der Forschung}

Schweiz Z Ganzheitsmed 2016;28:72-75

Published online: March 11, 2016

\title{
Die Behandlung menopausaler Beschwerden mit pflanzlichen Extrakten: Klinische Studien bestätigen die Wirksamkeit
}

\section{Cimicifuga racemosa}

Die Traubensilberkerze (Abb. 1) ist eine ausdauernde, krautige Pflanze, deren Heimat weite Gebiete Nordamerikas sind. Der heute korrekte botanische Name lautet Actea racemosa. In der Medizin wird aber immer noch der ursprüngliche Name Cimicifuga racemosa verwendet. Medizinisch werden die unterirdischen Teile, das Rhizom und die Wurzel eingesetzt.

Als Inhaltsstoffe konnten bisher Triterpenglykoside, Phenolsäuren, Flavonoide und weitere Substanzen isoliert werden. Diese entfalten eine Estrogen-ähnliche Wirkung und werden deshalb oft auch SERM (Selective Estrogen Receptor Modulators) genannt. Der immer wieder genannte Ausdruck «Phytoestrogene» könnte zu der Meinung führen, diese Naturstoffe seien Hormone oder hätten eine Hormonwirkung. Die Traubensilberkerze hat bzw. ihre Inhaltsstoffe haben mit Hormonen jedoch nichts

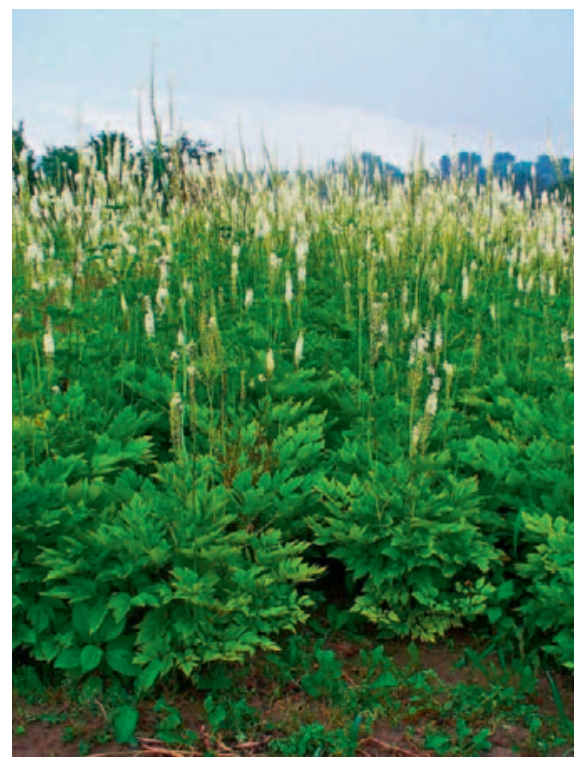

Abb. 1. Blühendes Feld von Traubensilberkerzen ( $\left.{ }^{(}\right)$H. Zell, 2009).

Tab. 1. Pflanzliche Präparate gegen menopausale Symptome

\begin{tabular}{|c|c|c|}
\hline Arzneipflanze & $\begin{array}{l}\text { Registrierte Präparate in } \\
\text { der Schweiz }\end{array}$ & Studienlage \\
\hline $\begin{array}{l}\text { Cimicifuga racemosa } \\
\quad \text { (Traubensilberkerze) }\end{array}$ & $\begin{array}{l}\text { Cimifemin }{ }^{\circledR} \\
\text { Cimifemin }^{\circledR} \text { forte, Cimifemin }{ }^{\circledR} \text { neo, } \\
\text { Cimifemin }{ }^{\circledR} \text { uno } \\
\text { Climavita }^{\circledR} \\
\text { Emoton Beta Cimicifuga }{ }^{\circledR} \\
\text { Femicin }^{\circledR} \\
\text { Feminelle }^{\circledR}\end{array}$ & $\begin{array}{l}\text { viele RCT mit positiven } \\
\text { Resultaten }\end{array}$ \\
\hline $\begin{array}{l}\text { Rheum rhaponticum } \\
\qquad \text { (Rhapontik-Rhabarber) }\end{array}$ & keine & $\begin{array}{l}\text { wenige RCT mit positiven } \\
\text { Resultaten }\end{array}$ \\
\hline $\begin{array}{l}\text { Isoflavone aus Soja oder } \\
\text { Trifolium pratense (Rotklee) }\end{array}$ & keine & $\begin{array}{l}\text { wenige Studien, zum Teil } \\
\text { positive Resultate }\end{array}$ \\
\hline Dioscorea villosa (Yamswurzel) & keine & keine klinischen Studien \\
\hline
\end{tabular}

\section{KARGER}

Fax +497614520714
() 2016 S. Karger GmbH, Freiburg 
zu tun; sie entfaltet eine andere Wirkung als Estrogene. Darum darf sie auch bei bestehendem Mammakarzinom bzw. im Falle eines familiären Risikos angewendet werden [1-3].

\section{Studien mit Traubensilberkerzen- Extrakten}

\section{Ze 450}

Die Studienlage über die Wirksamkeit von Cimicifuga ist wie erwähnt überzeugend.

In einer multizentrischen, offenen, prospektiven Therapiebeobachtung unter Praxisbedingungen mit 502 menopausalen Patientinnen wurde die Wirksamkeit eines standardisierten isopropanolischen Traubensilberkerzen-Extrakts während einer Dauer von 4 Monaten untersucht [4]. Dabei wurden typische menopausale Symptome wie Hitzewallungen, Schweissausbrüche, Verstimmungen, Schlafstörungen und Nervosität verglichen mit der Baseline signifikant verbessert ( $\mathrm{p}<0,0001$ ). 73,8\% der Patientinnen hatten von diesem Therapiekonzept einen positiven Eindruck.

\section{BNO 1055}

In einer von Raus et al. [5] publizierten Studie konnte neben der Wirksamkeit des standardisierten Traubensilberkerzen-Extrakts BNO 1055 auch die Sicherheit in Bezug auf die Veränderungen des Endometriums nachgewiesen werden.

Wuttke et al. [6] werteten eine 2003 publizierte Studie neu aus und konnten zeigen, dass der Cimicifuga-Extrakt klimakterische Beschwerden signifikant verbessert und ebenso wie eine HRT die Endometriumdicke nicht verändert sowie eine positive Wirkung auf das Vaginalepithelium und auf den Knochenmetabolismus hat.

\section{Beer, 2014}

Beer [7] publizierte 2014 einen Übersichtsartikel, in dem er auf die bisher erschienene Literatur einging. Darin erwähnt er 15 klinische Studien, die mit registrierten Cimicifuga-Präparaten durchgeführt wurden. Die beiden oben beschriebenen Studien (Ze 450, BNO 1055) sind in diesen 15 Studien inbegriffen.

Das Gesamtbild dieser 15 Studien liefert im Vergleich mit Placebo eine positive Evidenz für diese CimicifugaPräparate.

\section{Isoflavone}

Als weitere pflanzliche Zubereitung zur Linderung menopausaler Beschwerden werden auch Präparate angeboten, die reich an Isoflavonen sind (Abb. 2).

In der Natur kommen Isoflavone vor allem in Soja (Glycin-max-Familie der Hülsenfrüchtler) und im Rotklee (Trifolium pratense) vor [8].

\section{Studien mit Isoflavon-Präparaten}

\section{Chen et al., 2015}

Chen et al. [9] publizierten kürzlich eine Meta-Analyse über die Wirksamkeit von Isoflavon-haltigen Präparaten bei menopausalen Beschwerden und werteten 9 randomisierte kontrollierte Studien (RCT) aus. Bei 7 Studien wurde zur Wirksamkeitsbestimmung der Kupperman-Index verwendet. Bei diesen Studien wurde keine signifikante Wirksamkeit gefunden, jedoch wurde dies bei den 10 Studien mit der Anzahl Hitzewallungen als primärer Endpunkt festgestellt.

Diese Meta-Analyse birgt eine gewisse Unsicherheit, weil das Forscher-

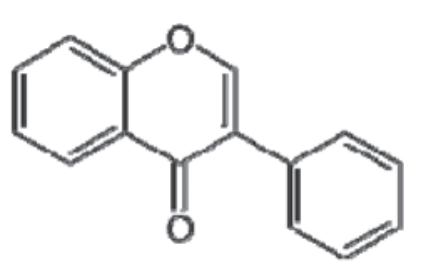

Abb. 2. Strukturformel von Isoflavon. team zur Auffindung von geeigneten Studien nicht nur das Stichwort «Isoflavone», sondern auch «Phytoestrogene» verwendet hatte, was eine gewisse Unsicherheit bezüglich der verwendeten Präparate mit sich bringt.

In der Schweiz gibt es keine registrierten Arzneimittel mit Isoflavonen, jedoch eine Reihe von Isoflavon-haltigen Nahrungsergänzungsmitteln.

\section{Coon et al., 2007}

Eine präzise Einschränkung zur Identifikation von geeigneten Studien machten Coon et al. [10] in einer 2007 publizierten Meta-Analyse, in die nur Studien eingeschlossen wurden, die mit Präparaten zur Behandlung von menopausalen Hitzewallungen durchgeführt wurden, deren Wirkstoff aus Rotklee gewonnene Isoflavone waren. 5 der 17 potenziell infrage gekommenen Studien entsprachen den Kriterien und konnten ausgewertet werden. Die Resultate dieser Studien zeigen Evidenz einer marginalen Verbesserung der Hitzewallungen.

\section{Rhapontik-Rhabarber}

Rhapontik-Rhabarber (Rheum rhaponticum), auch Sibirischer Rhabarber genannt, ist eine in nördlichen Gebieten der Nordhalbkugel wachsende Pflanze mit Hydroxystilbenen als Inhaltsstoffe. Beer [11] erwähnt in einem Übersichtsartikel 4 Studien, die mit dem Rhapontik-Rhabarber-Extrakt ERr 731 durchgeführt wurden. 3 davon, 2 RCT und eine Beobachtungsstudie, zeigten eine signifikante Verbesserung der menopausalen Beschwerden. In der Schweiz gibt es im Gegensatz zu Deutschland kein als Arzneimittel registriertes Präparat auf der Basis von R. rhaponticum.

\section{Yamswurzel}

Zur Behandlung menopausaler Beschwerden werden auch Zuberei- 
tungen in Form von Gel aus der Yamswurzel (Dioscorea villosa) eingesetzt. Es gibt aber keinerlei klinische Studien, welche die Wirksamkeit dieser Behandlungen dokumentieren. Anwender sind daher auf die Erfahrungen von Ärztinnen und Ärzten angewiesen, die Yamswurzel-Gel als Therapie vorschlagen. In der Schweiz kommt Yamswurzel als Nahrungsergänzungsmittel vor.

\section{Literatur}

1 Freudenstein J, et al: Lack of promotion of estrogenic-dependent mammary gland tumors in vivo by an isopropanolic Cimicifuga racemosa extract. Canc Res 2002;62:3448-3452.

$\checkmark 2$ Lupu R, et al: Black cohosh, a menopausal remedy, does not have estrogenic activity and does not promote breast cancer cell growth. Int J Oncol 2003;23:1407-1412.

$\checkmark 3$ Rostock M, et al: Black cohosh (Cimicifuga racemosa) in tamoxifen-treated breast cancer patients with climacteric complaints - a prospective observational study. Gynecol Endocrinol 2011;27:844-848.

4 Schmidt M, Polasek W, Käufeler R: Wirksamkeit und Sicherheit von Traubensilberkerze bei Menopausebeschwerden. J Menopause 2005; 12:30-34.

\section{Zusammenfassung}

Zur Behandlung von menopausalen Beschwerden steht als Alternative zur HRT eine Reihe von pflanzlichen Präparaten zur Verfügung. Die beste Studienlage weisen Präparate auf, deren Wirkstoff standardisierte Traubensilberkerzen-Extrakte sind. Davon sind in der Schweiz mehrere registrierte Arzneipräparate erhältlich. Sie eignen sich als relevante Alternative zur HRT für die Behandlung menopausaler Beschwerden.

Infrage kommen auch Präparate aus Rhapontik-Rhabarber oder mit Isoflavonen, deren Studienlage etwas weniger überzeugend ist als diejenige für die Traubensilberkerze. In der Schweiz stehen entsprechende Präparate jedoch nur als Nahrungsergänzungsmittel zur Verfügung. Dasselbe gilt für die Yamswurzel, zu der es keine klinischen Studien gibt.
5 Raus K, et al: First-time proof of endometrial safety of the special black cohosh extract (Actaea or Cimicifuga racemosa extract) CR BNO 1055. Menopause 2006;13:678-691.

6 Wuttke W, Seidlová-Wuttke D, Gorkow C: The Cimicifuga preparation BNO 1055 vs. conjugated estrogens in a double-blind placebo-controlled study: effects on menopause symptoms and bone markers. Maturitas 2003; 44(suppl 1):S67-77.

7 Beer A-M: Update Cimicifuga - neue Erkenntnisse aus Wissenschaft und Forschung: Differenzierte Evidenz für Wirksamkeit und Sicherheit von Traubensilberkerzen-Arzneimitteln zur Behandlung klimakterischer Beschwerden. J Gynäkol Endokrinol 2014;24:6-10.
8 Ghazanfarpour M, Sadeghi R, Roudsari RL, Khorsand I, Khadivzadeh T, Muoio B: Red clover for treatment of hot flashes and menopausal symptoms: a systematic review and meta-analysis. J Obstet Gynaecol 2015;DOI: 10.3109/01443615.2015.1049249.

9 Chen MN, et al: Efficacy of phytoestrogens for menopausal symptoms: a meta-analysis and systematic review. Climacteric 2015;18:260269.

10 Coon JT, Pittler MH, Ernst E: Trifolium pratense isoflavones in the treatment of menopausal hot flushes: a systematic review and metaanalysis. Phytomedicine 2007;14:53-59.

11 Beer A-M: Evidenz spricht für die Phytotherapie: Traubensilberkerze und Rhapontikrhabarber bei klimakterischen Beschwerden. MMW Fortschr Med 2014;156:40-41. 